Wright State University

CORE Scholar

6-1-2009

\title{
Spontaneously Generated Atomic Entanglement in Free Space Reinforced by Incoherent Pumping
}

Ling Zhou

Gou Hui Yang

Anil K. Patnaik

Wright State University - Main Campus, anil.patnaik@wright.edu

Follow this and additional works at: https://corescholar.libraries.wright.edu/physics

Part of the Physics Commons

\section{Repository Citation}

Zhou, L., Yang, G. H., \& Patnaik, A. K. (2009). Spontaneously Generated Atomic Entanglement in Free Space Reinforced by Incoherent Pumping. Physical Review A, 79 (6), 62102.

https://corescholar.libraries.wright.edu/physics/178

This Article is brought to you for free and open access by the Physics at CORE Scholar. It has been accepted for inclusion in Physics Faculty Publications by an authorized administrator of CORE Scholar. For more information, please contact library-corescholar@wright.edu. 


\title{
Spontaneously generated atomic entanglement in free space reinforced by incoherent pumping
}

\author{
Ling Zhou, ${ }^{1}$ Guo Hui Yang, ${ }^{1}$ and Anil K. Patnaik ${ }^{2,3, *}$ \\ ${ }^{1}$ School of Physics and Optoelectronic Technology, Dalian University of Technology, Dalian 116023, People's Republic of China \\ ${ }^{2}$ Department of Physics, Texas A\&M University, College Station, Texas 77843, USA \\ ${ }^{3}$ Department of Physics, Wright State University, Dayton, Ohio 45435, USA
}

(Received 26 March 2008; revised manuscript received 17 March 2009; published 5 June 2009)

\begin{abstract}
We study spontaneously generated entanglement (SGE) between two identical multilevel atoms in free space via vacuum-induced radiative coupling. We show that the SGE in two-atom systems may initially increase with time but eventually vanishes in the time scale determined by the excited-state lifetime and radiative coupling strength between the two atoms. We demonstrate that steady-state SGE can be established by incoherently pumping the atoms to their excited states. We have shown that an appropriate rate of incoherent pump can help in producing optimal steady-state SGE. The multilevel systems offer us more channels to establish entanglement. The system under consideration can be realized in a tight trap or atoms/ions doped in a solid substrate.

DOI: $10.1103 /$ PhysRevA.79.062102

PACS number(s): 03.65.Ud, 42.50.Dv
\end{abstract}

\section{INTRODUCTION}

The recent development of quantum technologies strives to resolve the quest for the best entanglement source in quantum optical systems. Although entanglement is observed in a variety of systems, entanglement in atomic systems are favored as more scalable and practical systems compared to their "photonic only" counterparts due to the development of reliable state-of-the-art technologies to control atoms one at a time [1] that can be precisely scaled to many atoms $[2,3]$. Many exciting developments of entanglement sources are based on atomic systems, e.g., entanglement via atom-cavity coupling [4], atom-atom entanglement via cavity [5], entanglement in trapped ions and atoms $[3,6]$, atomic entanglement in an optical lattice by controlled collision [7], and also atomic entanglement via external fields [8,9]. Recently, it has also been shown that well separated atomic ensembles can also be entangled via coherent coupling between them [10]. Scully [11] has extensively discussed entanglement in two, three, and many atoms via a single photon, and has shown that such an ensemble can produce directional spontaneous emission [12]. Das et al. [13] have shown that spatial variation in phase of an applied laser significantly alters the entanglement between two atoms.

Among the different atomic entanglement generation processes, an interesting and useful category is spontaneously generated entanglement (SGE) sources via interaction of atoms with a common bath of cavity field [5], vacuum $[3,6,14]$, heat bath $[15]$, or even a spin chain [16]. Usually, the baths have very short correlation time, and hence potentially cause disentanglement [17] and decoherence [18] in an entangled system. New studies of SGE in two two-level atoms in non-Markovian limit have been reported recently [19]. Effect of dipole-dipole $(d d)$ coupling on coherences in dense medium has been investigated [20]. Agarwal and Patnaik [21] have shown that coherences in two multilevel at-

\footnotetext{
*Present address: Air Force Research Laboratory, Propulsion Directorate, Wright-Patterson AFB, OH 45431 USA; anil.patnaik@wpafb.af.mil
}

oms can be generated from their interaction with a common vacuum bath via the retarded dipole-dipole coupling when they are brought to a close proximity of each other. Effect of such vacuum-induced coherence (VIC) on the collective resonance fluorescence is discussed in [22]. SGE is particularly interesting from the application point of view because practical quantum devices are often unavoidably coupled to the environmental bath and hence SGE can occur naturally.

Most of the works listed above are focused on SGE in two-level atoms. Study of multilevel systems is important because, in certain situations, participation of additional internal atomic levels in the process of generating entanglement or causing disentanglement is unavoidable. For example, when two atoms, having a triplet $P$ state as their excited [21] or ground [22] state, are placed in close proximity (i.e., the interatomic distance is less than the wavelength of the atomic transitions involved, $R<\lambda_{0}$ ), even the dipoles associated with orthogonal $\sigma_{ \pm}$transition moments can radiatively couple to generate additional coherences. Recently, Kiffner et al. [23] have explicitly shown that the twolevel approximation fails in such a situation. Furthermore, multilevel systems can open up new channels in bath assisted SGE in a very natural way and can provide us more control parameters [24]. To the best of our knowledge, only a few literature exist that addresses entanglement in three-level atoms interacting with a continuum via the radiative coupling $[17,24]$ where decoherence free subspace is investigated. However, to our knowledge a sustainable SGE in multilevel atomic systems has never been reported.

In this paper, we investigate the steady-state SGE between two radiatively coupled and incoherently pumped atoms having their energy levels in a $V$ configuration with nondegenerate excited states; see Fig. 1. We derive a master equation and trace over the field part to obtain the equations for the atomic dynamics. We are able to obtain an analytical solution to show that multilevel systems are preferable compared to two-level systems for SGE due to availability of additional coupling channels to establish the entanglement. We demonstrate that a sustainable steady-state entanglement can be achieved by purely incoherent processes. It may be noted that we are working outside the regime of VIC. In our twoatom system VIC could be generated if the excited states of 


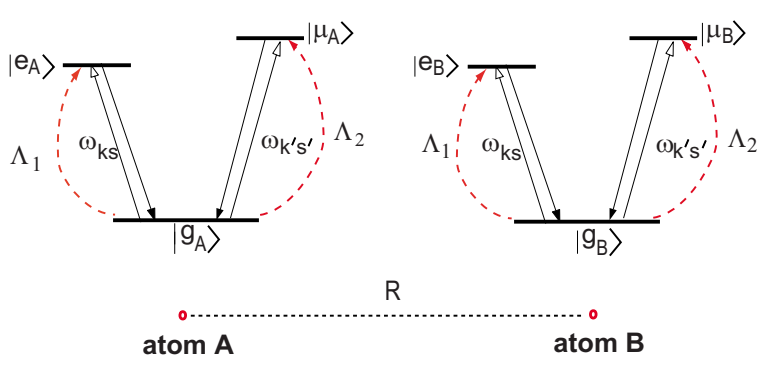

FIG. 1. (Color online) The two identical atoms under consideration. The $V$ type atoms with nondegenerate excited states. The distance between the two atoms is considered to be small compared to the radiation wavelength, $R<\lambda_{0}$.

both the atoms were degenerate or near degenerate $[21,22]$.

The organization of the paper is as follow: in Sec. II we derive a master equation for the two atoms interacting with the common vacuum, a reduced density-matrix equation that determines the system dynamics. In Sec. III, we present the time evolution of the entanglement between the two atoms that survives only for a short period of time. In Sec. IV, we derive the atomic density-matrix equations for incoherently pumped atoms. We show that a sustainable steady-state entanglement can be obtained between the two atoms purely via incoherent processes. We summarize and discuss our result in Sec. V.

\section{TWO-ATOM SYSTEM AND THEIR DYNAMICS}

We consider two identical three-level $V$ systems (say $A$ and $B)$ in free space having two excited states $\left|e_{\alpha}\right\rangle$ and $\left|\mu_{\alpha}\right\rangle$ $(\alpha=A, B)$, and a ground state $\left|g_{\alpha}\right\rangle$, as depicted in Fig. 1. Both the atoms couple to the same vacuum field. We do not wish to loose the generality of our results but to give an example, our scheme can correspond to two ${ }^{40} \mathrm{Ca}$ atoms in a magnetooptical trap in presence of a static magnetic field. The ground state can correspond to $4{ }^{1} S_{0}$ state and the excited states can correspond to the magnetic sublevels $4{ }^{1} P_{1}$ of $\mathrm{Ca}$ atom. The static magnetic field would remove the degeneracy of the $4{ }^{1} P_{1}$ sublevels. The states $\left|e_{\alpha}\right\rangle$ and $\left|\mu_{\alpha}\right\rangle$ can correspond to $m_{l}= \pm 1$ levels. Note that we restrict ourselves to a situation where the cross couplings between $\left|e_{\alpha}\right\rangle \leftrightarrow\left|g_{\alpha}\right\rangle$ and $\left|\mu_{\alpha}\right\rangle \leftrightarrow\left|g_{\alpha}\right\rangle$ transitions are eliminated by considering the nondegenerate excited states. Thus the photon emitted from $\left|e_{\alpha}\right\rangle \rightarrow\left|g_{\alpha}\right\rangle\left(\left|\mu_{\alpha}\right\rangle \rightarrow\left|g_{\alpha}\right\rangle\right)$ can only be absorbed by $\left|g_{\alpha}\right\rangle$ $\rightarrow\left|e_{\alpha}\right\rangle\left(\left|g_{\alpha}\right\rangle \rightarrow\left|\mu_{\alpha}\right\rangle\right)$. For simplicity, we consider only the case of real dipole moments for our discussion below. These results can be easily generalized to complex dipoles, e.g., involving magnetic sublevels.

In this section, we derive the system dynamics with only the contributions from the two atoms and vacuum coupling, i.e., in absence of incoherent pumping. The role of incoherent pumping will be discussed in detail in Sec. IV. The Hamiltonian of the two-atom system interacting with the vacuum field can be written in the interaction picture as

$$
\begin{aligned}
H_{I}= & \sum_{\alpha=A, B}\left(\sum_{k s} d_{e g}^{\alpha} \sigma_{e g}^{\alpha} a_{k s} e^{i\left[\vec{k} \cdot \vec{x}_{\alpha}+\left(\omega_{1}-\omega_{k s}\right) t\right]}\right. \\
& \left.+\sum_{k^{\prime} s^{\prime}} d_{\mu g}^{\alpha} \sigma_{\mu g}^{\alpha} a_{k^{\prime} s^{\prime}} e^{i\left[\vec{k}^{\prime} \cdot \vec{x}_{\alpha}+\left(\omega_{2}-\omega_{k^{\prime} s^{\prime}}\right) t\right]}+\text { H.c. }\right)
\end{aligned}
$$

where the vacuum Rabi coupling coefficients corresponding to atom $\alpha$ are

$$
\begin{gathered}
d_{e g}^{\alpha}=i\left(\frac{2 \pi \hbar \omega_{k s}}{V}\right)^{1 / 2} \vec{\wp}_{e g}^{\alpha} \cdot \vec{\varepsilon}_{k s}, \\
\text { and } d_{\mu g}^{\alpha}=i\left(\frac{2 \pi \hbar \omega_{k^{\prime} s^{\prime}}}{V}\right)^{1 / 2} \vec{\wp}_{\mu g}^{\alpha} \cdot \vec{\varepsilon}_{k^{\prime} s^{\prime}},
\end{gathered}
$$

and $\vec{\wp}_{e g}^{\alpha}\left(\vec{\wp}_{\mu g}^{\alpha}\right)$ is the dipole matrix element corresponding to the transition operator $\sigma_{e g}^{\alpha}=\left|e_{\alpha}\right\rangle\left\langle g_{\alpha}\right|\left(\sigma_{\mu g}^{\alpha}=\left|\mu_{\alpha}\right\rangle\left\langle g_{\alpha}\right|\right), \vec{\varepsilon}_{k s}\left(\vec{\varepsilon}_{k^{\prime} s^{\prime}}\right)$ is the unit polarization vector of the vacuum mode with frequency $\omega_{k s}\left(\omega_{k^{\prime} s^{\prime}}\right), a_{k s}\left(a_{k^{\prime} s^{\prime}}\right)$ is the photon annihilation operator corresponding to the vacuum field with wave vector $k\left(k^{\prime}\right)$ and polarization $s\left(s^{\prime}\right), \omega_{1}\left(\omega_{2}\right)$ is the atomic frequency corresponding to $\left|e_{\alpha}\right\rangle \leftrightarrow\left|g_{\alpha}\right\rangle\left(\left|\mu_{\alpha}\right\rangle \leftrightarrow\left|g_{\alpha}\right\rangle\right)$ transitions, and $x_{\alpha}$ is the position of the atom $\alpha$.

We use the Zwanzig projection operator method $[25,26]$ to trace over the field degrees of freedom and obtain a reduced density-matrix equation for the atoms. We use the Born and Markoff approximation to obtain a memoryless master equation. Referring to Ref. [21] and without duplicating the lengthy calculation, we write the reduced densitymatrix equation for the atoms as

$$
\dot{\rho}=-i\left[\mathcal{V}_{d d}, \rho\right]+\left(\mathcal{L}_{s}+\mathcal{L}_{d d}\right) \rho,
$$

where

$$
\mathcal{V}_{d d}=G_{1} \sigma_{e g}^{A} \otimes \sigma_{g e}^{B}+G_{2} \sigma_{\mu g}^{A} \otimes \sigma_{g \mu}^{B}+\text { H.c. }
$$

is the part of the $d d$ interaction that contributes to the level shift. The coupling coefficients are

$$
\begin{aligned}
& G_{1}=\sum_{k s} \frac{\pi}{\hbar^{2}\left(\omega_{1}-\omega_{k s}\right)} d_{e g}^{A} d_{g e}^{B} e^{i \vec{k} \cdot \vec{R}}, \\
& G_{2}=\sum_{q l} \frac{\pi}{\hbar^{2}\left(\omega_{2}-\omega_{q l}\right)} d_{\mu g}^{A} d_{g \mu}^{B} e^{i \vec{k} \cdot \vec{R}} .
\end{aligned}
$$

Here $\vec{R}=\vec{x}_{A}-\vec{x}_{B}$. Further, the Liouvillian operators $\mathcal{L}_{j}$ are

$$
\begin{aligned}
\mathcal{L}_{s} \rho= & \gamma_{1}\left[\left(2 \sigma_{g e}^{A} \rho \sigma_{e g}^{A}-\sigma_{e e}^{A} \rho-\rho \sigma_{e e}^{A}\right)+A \rightarrow B\right]+\gamma_{2}\left[\left(2 \sigma_{g \mu}^{A} \rho \sigma_{\mu g}^{A}\right.\right. \\
& \left.\left.-\sigma_{\mu \mu}^{A} \rho-\rho \sigma_{\mu \mu}^{A}\right)+A \rightarrow B\right],
\end{aligned}
$$

corresponding to spontaneous emission of the atoms, and

$$
\begin{aligned}
\mathcal{L}_{d d} \rho= & {\left[\Gamma_{1}\left(2 \sigma_{g e}^{B} \rho \sigma_{e g}^{A}-\sigma_{e g}^{A} \sigma_{g e}^{B} \rho-\rho \sigma_{e g}^{A} \sigma_{g e}^{B}\right)+\text { H.c. }\right] } \\
& +\left[\Gamma_{2}\left(2 \sigma_{g \mu}^{B} \rho \sigma_{\mu g}^{A}-\sigma_{\mu g}^{A} \sigma_{g \mu}^{B} \rho-\rho \sigma_{\mu g}^{A} \sigma_{g \mu}^{B}\right)+\text { H.c. }\right],
\end{aligned}
$$

corresponding to the $d d$ coupling mediated by the vacuum. Note that the subscript in $\rho_{a}$ is dropped for brevity. Here the spontaneous decay rates are given as 


$$
\begin{aligned}
& \gamma_{1}=\frac{1}{\hbar^{2}} \sum_{k s} \pi \delta\left(\omega_{1}-\omega_{k s}\right)\left|d_{e g}\right|^{2}, \\
& \gamma_{2}=\frac{1}{\hbar^{2}} \sum_{q l} \pi \delta\left(\omega_{2}-\omega_{q l}\right)\left|d_{\mu g}\right|^{2},
\end{aligned}
$$

and the atom-atom coupling coefficients are obtained as

$$
\begin{aligned}
& \Gamma_{1}=\frac{1}{\hbar^{2}} \sum_{k s} \pi \delta\left(\omega_{1}-\omega_{k s}\right)\left|d_{e g}\right|^{2} e^{i \vec{k} \cdot \vec{R}}, \\
& \Gamma_{2}=\frac{1}{\hbar^{2}} \sum_{k s} \pi \delta\left(\omega_{2}-\omega_{q l}\right)\left|d_{\mu g}\right|^{2} e^{i \vec{k} \cdot \vec{R}} .
\end{aligned}
$$

Further the index $\alpha$ has been dropped as we consider that the atomic dipoles corresponding to the same atomic transitions are parallel to each other, i.e., $\vec{\wp}_{i j}^{A} \| \vec{\wp}_{i j}^{B}$. Clearly, the radiative coupling terms $\Gamma_{i}$ and $G_{i}$ have numerical significance only in the limit $|k R|$ and $\left|k^{\prime} R\right| \leq 1$. In the other limit, when the interatomic distance $R$ is too large, only the spontaneous emission terms survive and the atoms behave as two independent and uncorrelated systems. We refer to [21] for the detailed steps of the calculation.

Now let us assume that initially the two-atom state is $|e \mu\rangle$, where we use the notation $|i j\rangle \equiv\left|i_{A}\right\rangle \otimes\left|j_{B}\right\rangle, i, j=e, \mu, g$. The nine two-atom basis states are $|e e\rangle,|e \mu\rangle,|e g\rangle,|\mu e\rangle$, $|\mu \mu\rangle,|\mu g\rangle,|g e\rangle,|g \mu\rangle$, and $|g g\rangle$, and we number them one through nine in the same order as above to simplify the notations for the density-matrix elements $\left\langle i_{A} j_{B}|\rho| i_{A}^{\prime} j_{B}^{\prime}\right\rangle$. For example, the density-matrix element $\left\langle e_{A} \mu_{B}|\rho| e_{A} \mu_{B}\right\rangle$ corresponding to our initial state $|e \mu\rangle$ is represented as $\rho_{22}$ in the above notation. The full density-matrix equation involves 81 matrix elements but for the above initial condition, many matrix elements are identically zero and only ten densitymatrix elements survive. We consider the geometry where dipole matrix elements are orthogonal to each other and are real (corresponding to real dipole moments as discussed in Sec. III of [21]), such that the parameters $G_{i}, \gamma_{i}$, and $\Gamma_{i}$ are real numbers. In the following, we explicitly write the dynamics equations only for those surviving density-matrix elements as

$$
\begin{gathered}
\dot{\rho}_{22}=-2\left(\gamma_{1}+\gamma_{2}\right) \rho_{22}, \\
\dot{\rho}_{33}=-2 \gamma_{1} \rho_{33}+2 \gamma_{2} \rho_{22}-\Gamma_{1}\left(\rho_{73}+\rho_{37}\right)-i G_{1}\left(\rho_{73}-\rho_{37}\right), \\
\dot{\rho}_{37}=-2 \gamma_{1} \rho_{37}-\Gamma_{1}\left(\rho_{77}+\rho_{33}\right)-i G_{1}\left(\rho_{77}-\rho_{33}\right), \\
\dot{\rho}_{66}=-2 \gamma_{2} \rho_{66}-\Gamma_{2}\left(\rho_{86}+\rho_{68}\right)-i G_{2}\left(\rho_{86}-\rho_{68}\right), \\
\dot{\rho}_{68}=-2 \gamma_{2} \rho_{68}-\Gamma_{2}\left(\rho_{88}+\rho_{66}\right)-i G_{2}\left(\rho_{88}-\rho_{66}\right), \\
\dot{\rho}_{77}=-2 \gamma_{1} \rho_{77}-\Gamma_{1}\left(\rho_{37}+\rho_{73}\right)-i G_{1}\left(\rho_{37}-\rho_{73}\right), \\
\dot{\rho}_{88}=-2 \gamma_{2} \rho_{88}+2 \gamma_{1} \rho_{22}-\Gamma_{2}\left(\rho_{86}+\rho_{68}\right)-i G_{2}\left(\rho_{68}-\rho_{86}\right),
\end{gathered}
$$

$$
\begin{aligned}
\dot{\rho}_{99}= & 2 \gamma_{1}\left(\rho_{33}+\rho_{77}\right)+2 \gamma_{2}\left(\rho_{66}+\rho_{88}\right)+2 \Gamma_{1}\left(\rho_{37}+\rho_{73}\right) \\
& +2 \Gamma_{2}\left(\rho_{68}+\rho_{86}\right) .
\end{aligned}
$$

Note that the conjugate matrix elements $\rho_{73}$ and $\rho_{86}$ (conjugates of $\rho_{37}$ and $\rho_{68}$, respectively) also evolve. Using the Laplace transform method, we solve the above coupled equations for the density-matrix elements with the initial condition $\rho_{22}=1$ to obtain their time evolution as

$$
\begin{aligned}
& \rho_{22}(t)=e^{-2\left(\gamma_{1}+\gamma_{2}\right) t}, \\
& \rho_{33}(t)=\frac{\gamma_{2} e^{-2 \gamma_{1} t}}{2} \\
& \times\left[\frac{\gamma_{2} \cosh \left(2 \Gamma_{1} t\right)-\Gamma_{1} \sinh \left(2 \Gamma_{1} t\right)-\gamma_{2} e^{-2 \gamma_{2} t}}{\gamma_{2}^{2}-\Gamma_{1}^{2}}\right. \\
& \left.+\frac{\gamma_{2} \cos \left(2 G_{1} t\right)+G_{1} \sin \left(2 G_{1} t\right)-\gamma_{2} e^{-2 \gamma_{2} t}}{\gamma_{2}^{2}+G_{1}^{2}}\right] \text {, } \\
& \rho_{37}(t)=\frac{\gamma_{2} e^{-2 \gamma_{1} t}}{2} \\
& \times\left[\frac{\Gamma_{1} e^{-2 \gamma_{2} t}-\Gamma_{1} \cosh \left(2 \Gamma_{1} t\right)+\gamma_{2} \sinh \left(2 \Gamma_{1} t\right)}{\Gamma_{1}^{2}-\gamma_{2}^{2}}\right. \\
& \left.-\frac{i G_{1} \cos \left(2 G_{1} t\right)-i \gamma_{2} \sin \left(2 G_{1} t\right)-i G_{1} e^{-2 \gamma_{2} t}}{G_{1}^{2}+\gamma_{2}^{2}}\right] \text {, } \\
& \rho_{66}(t)=\frac{\gamma_{1} e^{-2 \gamma_{2} t}}{2} \\
& \times\left[\frac{\gamma_{1} \cosh \left(2 \Gamma_{2} t\right)-\Gamma_{2} \sinh \left(2 \Gamma_{2} t\right)-\gamma_{1} e^{-2 \gamma_{1} t}}{\gamma_{1}^{2}-\Gamma_{2}^{2}}\right. \\
& \left.-\frac{\gamma_{1} \cos \left(2 G_{2} t\right)+G_{2} \sin \left(2 G_{2} t\right)-\gamma_{1} e^{-2 \gamma_{1} t}}{\gamma_{1}^{2}+G_{2}^{2}}\right] \text {, } \\
& \rho_{68}(t)=\left.\rho_{37}^{*}(t)\right|_{1 \leftrightarrow 2}, \rho_{77}(t)=\left.\rho_{66}(t)\right|_{1 \leftrightarrow 2}, \\
& \rho_{88}(t)=\left.\rho_{33}(t)\right|_{1 \leftrightarrow 2}, \\
& \rho_{99}(t)=1-\rho_{22}-\rho_{33}(t)-\rho_{66}(t)-\rho_{77}(t)-\rho_{88}(t) .
\end{aligned}
$$

It may be noted that the initial state $\rho_{22} \equiv\left\langle e_{A} \mu_{B}|\rho| e_{A} \mu_{B}\right\rangle$ decays with a rate of the sum of the decays of both excited states but does not depend on the $d d$ coupling terms $\Gamma_{i}$ and $G_{i}$. However, the other population and cross terms strongly depend on the $d d$ coupling. The coupling coefficient $\Gamma_{i}$ represents decay of the two-atom system via the cosine and sine hyperbolic functions, and $G_{i}$ is the $d d$ coupling induced vacuum Rabi oscillations. The time dependent solutions of the matrix elements show the oscillations with frequencies determined by the atom-atom coupling coefficients $G_{1}$ and $G_{2}$. Furthermore, the $d d$ couplings are strongly dependent on the interatomic distance $R$. Hence the dynamics of the density-matrix elements are also strongly affected by $R$. In the following section, we will present numerical plots and discussions for some of the important density-matrix elements that help in generating the SGE. 


\section{TIME EVOLUTION OF SGE}

In this section, we calculate the time evolution of the entanglement between two atoms. Out of various different methods to calculate entanglement between the two atoms, we choose the negativity, defined by [27]

$$
N(\rho)=\frac{\left\|\rho^{T_{A}}\right\|-1}{2}=-\sum_{i}{ }^{\prime} \lambda_{i},
$$

as the measure of entanglement. Here $\left\|\rho^{T_{A}}\right\|$ denotes the trace norm of $\rho^{T_{A}}$ [28]; $\rho^{T_{A}}$ is the partial transposition matrix of the atomic system density operator $\rho(t)$. The primed sum in the above equation represents the sum over only the negative eigenvalues $\lambda_{i}$ of $\rho^{T_{A}}$. For a high dimensional system, while a nonzero $N(\rho)$ is a sufficient condition to prove that a system is entangled, a null $N(\rho)$ does not necessarily confirm that the system is not entangled [29]. However, in the following we will show that for our system, $N(\rho) \neq 0$ is both necessary and sufficient condition for entanglement. From the definition, the negativity $N$ can also be greater than unity. For different dimensions of the density matrix, the maximum value of $N$ is different. For a two-atom three-level system, such as ours, the state $\Psi=\frac{1}{\sqrt{3}}[|e e\rangle+|\mu \mu\rangle+|g g\rangle]$ is maximally entangled one with the negativity $N(\Psi)=1$.

To obtain negativity in our two-atom system, we first calculate the eigenvalues $\lambda_{i}$ of $\rho^{T_{A}}$ by using the density matrix $\rho(t)$. After a lengthy calculation, we obtain the exact eigenvalues as

$$
\begin{gathered}
\lambda_{1}=\lambda_{2}=0, \quad \lambda_{3}=\rho_{22}(t), \quad \lambda_{4}=\rho_{33}(t), \\
\lambda_{5}=\rho_{66}(t), \quad \lambda_{6}=\rho_{77}(t), \quad \lambda_{7}=\rho_{88}(t), \\
\lambda_{8}=\frac{\rho_{99}(t)}{2}+\frac{1}{2} \sqrt{\rho_{99}^{2}(t)+4\left[\left|\rho_{37}(t)\right|^{2}+\left|\rho_{68}(t)\right|^{2}\right]}, \\
\lambda_{9}=\frac{\rho_{99}(t)}{2}-\frac{1}{2} \sqrt{\rho_{99}^{2}(t)+4\left[\left|\rho_{37}(t)\right|^{2}+\left|\rho_{68}(t)\right|^{2}\right]} .
\end{gathered}
$$

Among all of the above eigenvalues, only $\lambda_{9}$ can become negative. Clearly, for $\lambda_{9}$ to be negative, at least one of the matrix element $\rho_{37} \equiv\langle e g|\rho| g e\rangle$ or $\rho_{68} \equiv\langle\mu g|\rho| g \mu\rangle$ should be nonzero, i.e., there should be exchange in at least one photon between two atoms.

Entanglement between the two atoms can be created only if they interact. Therefore, if there is no photon-exchange interaction between the two atoms, there is no entanglement in the system. Mathematically, if all off-diagonal elements are identically zero, $\left(\rho_{37}=\rho_{68}=0\right)$, from Eq. (13), $\lambda_{9}=0$ and hence $N(\rho)=0$, then there is no entanglement in the system. However, if any one of the above photon-exchange interaction occurs, then $\lambda_{9}$ becomes negative and hence $N(\rho) \neq 0$, which is a sufficient condition to prove occurrence of SGE. Therefore, nonzero negativity is both a necessary and sufficient condition for entanglement in the dynamics of our twoatom model, unlike the general case in [29]. Hence negative $\lambda_{9}$ is a good measure of entanglement in our system. Note that, both $\rho_{37}$ and $\rho_{68}$ contribute to the generation of entanglement, thus the three-level atoms offer us more chan-
TABLE I. The interatomic distance and the corresponding coupling parameters. All the frequency units are scaled with $\gamma$.

\begin{tabular}{lllll}
\hline \hline$R$ & & & & \\
$\left(\right.$ in unit of $\left.\lambda_{0}\right)$ & $\Gamma_{1}$ & $\Gamma_{2}$ & $G_{1}$ & $G_{2}$ \\
\hline 0.50 & $0.96 \gamma_{1}$ & $0.96 \gamma_{2}$ & $8.0 \gamma_{1}$ & $8.0 \gamma_{2}$ \\
0.83 & $0.9 \gamma_{1}$ & $0.9 \gamma_{2}$ & $2.4 \gamma_{1}$ & $2.4 \gamma_{2}$ \\
1.18 & $0.8 \gamma_{1}$ & $0.8 \gamma_{2}$ & $0.9 \gamma_{1}$ & $0.9 \gamma_{2}$ \\
2.78 & $0.2 \gamma_{1}$ & $0.2 \gamma_{2}$ & $-0.24 \gamma_{1}$ & $-0.24 \gamma_{2}$ \\
\hline \hline
\end{tabular}

nels to establish entanglement than two-level atoms.

Before we discuss evolution of SGE, we present study of the density-matrix elements $\rho_{37}(t)$ and $\rho_{99}(t)$ that determine the entanglement. Note that the two excited states are nondegenerate. Assuming that $\omega_{2}=r \omega_{1}$, and say $\gamma_{1}=\gamma, \Gamma_{1}=\Gamma$, $G_{1}=G$, we have $\gamma_{2}=r \gamma, \Gamma_{2}=r \Gamma$, and $G_{2}=r G$. For the numerical plots presented below, we use the parameters given in Table I determined from their definitions in Eqs. (5), (8), and (9) [30]. Also note that both $\Gamma_{i}$ and $G_{i}$ oscillate with the interatomic distance [21]. However, peak value of $\Gamma_{i}\left(G_{i}\right)$ decreases with as interatomic distance decreases due to reduced dipole-dipole coupling between the two atoms. Thus, $\Gamma_{i}\left(G_{i}\right)$ can have same value in two or more values interatomic distances. But for a given interatomic distance, the value of the pair $\left(\Gamma_{i}, G_{i}\right)$ determine the effective $d d$ coupling. We will concentrate on the photon-exchange process for a decreased trend of $\Gamma_{i}\left(G_{i}\right)$ with an increased interatomic separation, such that the parameters given in Table I are approximately monotonic.

Using the above set of parameters, we present the evolution of the density-matrix element $\rho_{37}=\langle e g|\rho| g e\rangle$ representing the single-photon radiative coupling process in Fig. 2. Here, atom $A$ loses its excitation to excite atom $B$ from its ground state $\left|g_{A}\right\rangle$ to the state $\left|e_{B}\right\rangle$. It is observed that initially the number of photon-exchange events increase with time.

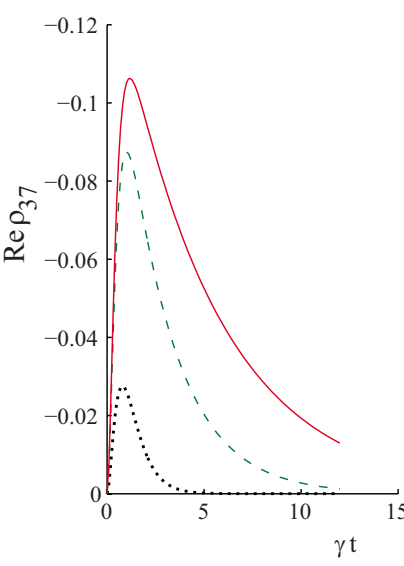

(a)

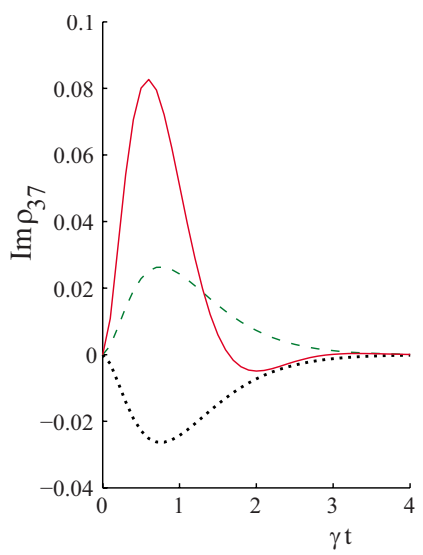

(b)
FIG. 2. (Color online) The time dependence of real and imaginary parts of matrix element $\rho_{37}(t)$ with different values of $\Gamma_{1}\left(\Gamma_{2}\right)$ and $G_{1}\left(G_{2}\right)$. We set $\gamma=1, \quad r=1.2$. The parameters $(G, \Gamma)$ $\equiv(2.4,0.9),(0.9,0.8)$, and $(-0.24,0.2)$ correspond to $R=0.83 \lambda_{1}$, $1.18 \lambda_{1}$, and $2.78 \lambda_{1}$ that are represented in the figure as the solid, dashed, and dotted lines, respectively. 


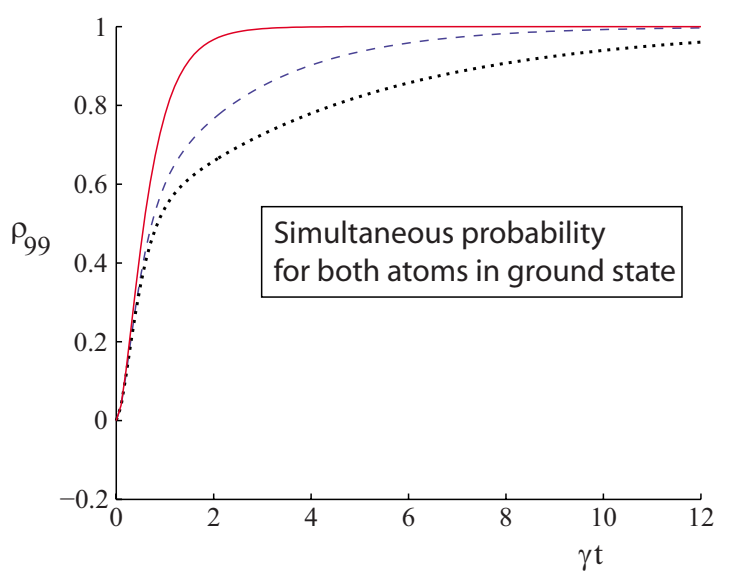

FIG. 3. (Color online) The matrix element for our atomic system as a function of $t$ corresponding to different pairs of $(G, \Gamma)$. The curves and also corresponding legends are the same as in Fig. 2.

However, after reaching a maximum, $\operatorname{Re} \rho_{37}$ falls off quickly within one spontaneous emission cycle. The time needed to reach the maximum value for $\operatorname{Re} \rho_{37}$ is determined by $\Gamma^{-1}$. The maximum of $\operatorname{Im} \rho_{37}$ occurs at $t \sim \gamma^{-1}$. In long-time limit both real and imaginary parts of $\rho_{37}$ vanish. Similar conclusions can be derived for the matrix element $\rho_{68}$ which physically represents the simultaneous probability of two processes $\left|\mu_{A}\right\rangle \rightarrow\left|g_{A}\right\rangle$ and $\left|g_{B}\right\rangle \rightarrow\left|\mu_{B}\right\rangle$.

In Fig. 3 we present the evolution of the population in the state $|g g\rangle$, i.e., the matrix element $\rho_{99}(t)$. This plot also supports the physical process we described above. We can see that again large values of $\Gamma$ slows down $\rho_{99}(t)$ to reach at its asymptotic value. In other words, the radiative coupling process survives longer due to the enhanced photon-exchange process with stronger $d d$ coupling strength. The steady-state value is $\rho_{99}=1$, i.e., both the atoms reach their ground states in the long-time limit.

Next, we discuss the property of the entanglement generated in this atom pair. We present the plot of $N(t)$ that describes the time evolution of the SGE for different values of $\Gamma$ and $G$ in Fig. 4. It is shown that at $t=0$, there is no entanglement because initially the atomic system is in the state $|e \mu\rangle$. For $t>0, N(t)$ evolves to reach its maximum value, and then undergoes a process of disentanglement. Finally, steady state of negativity becomes identically zero. But the relaxation time for $N$ becomes longer with smaller interatomic distances for our chosen parameters. From the solution [Eq. (11)], we know that relaxation time of $\operatorname{Re}\left(\rho_{37}\right)$ is determined by $\max \left\{\frac{1}{2\left(\gamma_{1}+\Gamma_{1}\right)}, \frac{1}{2\left(\gamma_{1}-\Gamma_{1}\right)}\right\}$ which means that the larger the value of $\left|\Gamma_{1}\right|$, the longer the relaxation time for disentanglement. Note that the relaxation time is determined by two competing radiative processes: the photon exchange between the atoms that leads to SGE and the loss of photon due to spontaneous emission that occurs in all $4 \pi$ direction. The evolution of SGE can be understood as due to the following multiple radiative exchange processes: spontaneous emission of atom $A$ via $\left|e_{A}\right\rangle \rightarrow\left|g_{A}\right\rangle$ (atom $B$ via $\left|\mu_{B}\right\rangle \rightarrow\left|g_{B}\right\rangle$ ) transition is followed by an absorption of the spontaneously emitted radiation by atom $B$ via $\left|g_{B}\right\rangle \rightarrow\left|e_{B}\right\rangle$ (atom $A$ via $\left|g_{A}\right\rangle \rightarrow\left|\mu_{A}\right\rangle$ ), as it is expressed by $\rho_{37}(t)\left[\rho_{68}(t)\right]$ causing the two-atom entanglement.

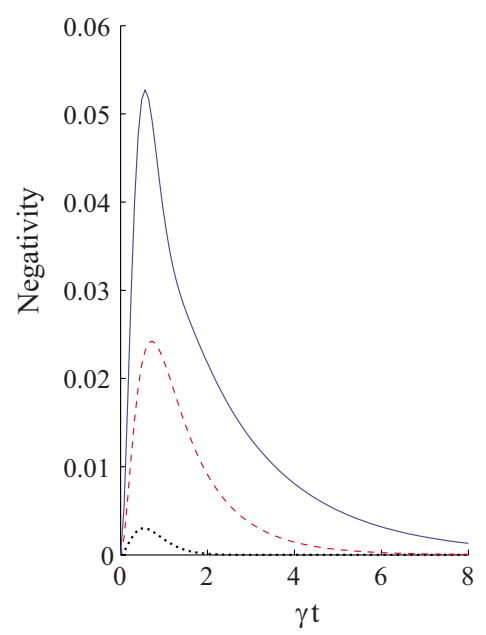

FIG. 4. (Color online) The negativity for our atomic system as a function of $t$ corresponding to different pairs of $(G, \Gamma)$. All the parameters correspond to Fig. 2, respectively.

\section{STEADY STATE ENTANGLEMENT WITH BROADBAND INCOHERENT PUMPING}

From the previous section, we have seen that the disadvantage of SGE is: although SGE evolves in time due to $d d$ coupling, it quickly diminishes due to the spontaneous decays in the system. However, for any useful application, for example, to use the two-atom system as a coupled qubit, sustaining the generated SGE is essential. In order to achieve a steady-state entanglement, we introduce an incoherent pump to continually repump population to the excited states as shown in Fig. 1. Steady state entanglement between two two-level atoms using a monochromatic coherent pumping is discussed in [31]. Coherent field assisted entanglement is rather intuitive. On the contrary, an incoherent pumping would be usually associated with decoherence and, hence, disentanglement in the system. However, in what follows below, we show that an appropriate strength of incoherent pump can lead to a steady entanglement between the two atoms, without requiring any additional atomic coherences, unlike in Ref. [31]. The SGE described below is generated purely by incoherent processes.

We consider a broadband incoherent pump acting on the two atoms which incoherently drive the population from $|g\rangle$ to $|e\rangle$ and $|\mu\rangle$ levels. Incoherent pumping can be modeled as an inverse process of spontaneous emission $[32,33]$. Thus we add a third Liouvillian $\mathcal{L}_{\text {inc }}$ to the master equation [Eq. (4)] to get

$$
\dot{\rho}=-i\left[\mathcal{V}_{d d}, \rho\right]+\left(\mathcal{L}_{s}+\mathcal{L}_{d d}+\mathcal{L}_{\text {inc }}\right) \rho,
$$

where

$$
\begin{aligned}
\mathcal{L}_{\text {inc }} \rho= & \Lambda_{1}\left[\left(2 \sigma_{e g}^{A} \rho \sigma_{g e}^{A}-\sigma_{g g}^{A} \rho-\rho \sigma_{g g}^{A}\right)+A \rightarrow B\right] \\
& +\Lambda_{2}\left[\left(2 \sigma_{\mu g}^{A} \rho \sigma_{g \mu}^{A}-\sigma_{\mu \mu}^{A} \rho-\rho \sigma_{\mu \mu}^{A}\right)+A \rightarrow B\right],
\end{aligned}
$$

where $\Lambda_{1}$ and $\Lambda_{2}$ denote incoherent pumping rates for $\left|g_{\alpha}\right\rangle$ $\rightarrow\left|e_{\alpha}\right\rangle$ and $\left|g_{\alpha}\right\rangle \rightarrow\left|\mu_{\alpha}\right\rangle$ transitions, respectively. We explicitly 
write the density-matrix equations involved in presence of the incoherent pump as

$$
\begin{aligned}
& \dot{\rho}_{11}=-4 \gamma_{1} \rho_{11}+2 \Lambda_{1}\left(\rho_{77}+\rho_{33}\right), \\
& \dot{\rho}_{22}=-2\left(\gamma_{1}+\gamma_{2}\right) \rho_{22}+2 \Lambda_{1} \rho_{88}+2 \Lambda_{2} \rho_{33}, \\
& \dot{\rho}_{33}=-2 s_{1} \rho_{33}+2 \gamma_{2} \rho_{22}+2 \gamma_{1} \rho_{11}+2 \Lambda_{1} \rho_{99}-i G_{1}\left(\rho_{73}-\rho_{37}\right) \\
& -\Gamma_{1}\left(\rho_{73}+\rho_{37}\right) \text {, } \\
& \dot{\rho}_{37}=-2 s_{1} \rho_{37}-\Gamma_{1}\left(\rho_{77}+\rho_{33}\right)+2 \Gamma_{1} \rho_{11}-i G_{1}\left(\rho_{77}-\rho_{33}\right), \\
& \dot{\rho}_{44}=-2\left(\gamma_{1}+\gamma_{2}\right) \rho_{44}+2 \Lambda_{1} \rho_{66}+2 \Lambda_{2} \rho_{77}, \\
& \dot{\rho}_{55}=-4 \gamma_{2} \rho_{55}+2 \Lambda_{2}\left(\rho_{66}+\rho_{88}\right), \\
& \dot{\rho}_{66}=-2 s_{2} \rho_{66}+2 \gamma_{1} \rho_{44}+2 \gamma_{2} \rho_{55}+2 \Lambda_{2} \rho_{99}-i G_{2}\left(\rho_{86}-\rho_{68}\right) \\
& -\Gamma_{2}\left(\rho_{86}+\rho_{68}\right) \text {, } \\
& \dot{\rho}_{68}=-2 s_{2} \rho_{68}-\Gamma_{2}\left(\rho_{88}+\rho_{66}\right)+2 \Gamma_{1} \rho_{55}-i G_{2}\left(\rho_{88}-\rho_{66}\right), \\
& \dot{\rho}_{77}=-2 s_{1} \rho_{77}+2 \gamma_{1} \rho_{11}+2 \gamma_{2} \rho_{44}+2 \Lambda_{1} \rho_{99}-\Gamma_{1}\left(\rho_{37}+\rho_{73}\right) \\
& -i G_{1}\left(\rho_{37}-\rho_{73}\right) \text {, } \\
& \dot{\rho}_{88}=-2 s_{2} \rho_{88}+2 \gamma_{1} \rho_{22}+2 \gamma_{2} \rho_{55}+2 \Lambda_{2} \rho_{99}-\Gamma_{2}\left(\rho_{86}+\rho_{68}\right) \\
& -i G_{2}\left(\rho_{68}-\rho_{86}\right) \text {, } \\
& \dot{\rho}_{99}=2 \gamma_{1}\left(\rho_{33}+\rho_{77}\right)+2 \gamma_{2}\left(\rho_{66}+\rho_{88}\right)-4\left(\Lambda_{1}+\Lambda_{2}\right) \rho_{99} \\
& +2 \Gamma_{1}\left(\rho_{37}+\rho_{73}\right)+2 \Gamma_{2}\left(\rho_{68}+\rho_{86}\right) \text {. }
\end{aligned}
$$

where $s_{\alpha}=\gamma_{\alpha}+\Lambda_{1}+\Lambda_{2}(\alpha=1,2)$. Note that in presence of the incoherent pumping $\Lambda_{i}$, the nonzero additional terms are the populations $|e e\rangle,|\mu \mu\rangle$, and $|\mu e\rangle$. Hence we have a total of 13 nonvanishing density-matrix elements in presence of $\Lambda_{i}$. Since we are looking for a steady-state SGE, we calculate the steady-state values of the matrix elements by setting the differentials in the left-hand sides of Eqs. (16) to zero and solving the coupled linear equations. The analytical solutions that we obtain are

$$
\begin{gathered}
\rho_{11}=\frac{\gamma_{2}}{b} \Lambda_{1} a_{2}, \\
\rho_{22}=\frac{\gamma_{1} \gamma_{2}}{b\left(\gamma_{1}+\gamma_{2}\right)}\left(a_{1} \Lambda_{1}+a_{2} \Lambda_{2}\right), \\
\rho_{33}=\frac{\gamma_{1} \gamma_{2}}{b} a_{2}, \\
\rho_{37}=\frac{\Gamma_{1} \gamma_{2}}{s_{1} b}\left(\Lambda_{1}-\gamma_{1}\right) a_{2}, \quad \rho_{44}=\rho_{22}, \\
\rho_{55}=\frac{\gamma_{1} a_{1}}{b} \Lambda_{2}, \quad \rho_{66}=\frac{\gamma_{1} \gamma_{2} a_{1}}{b},
\end{gathered}
$$

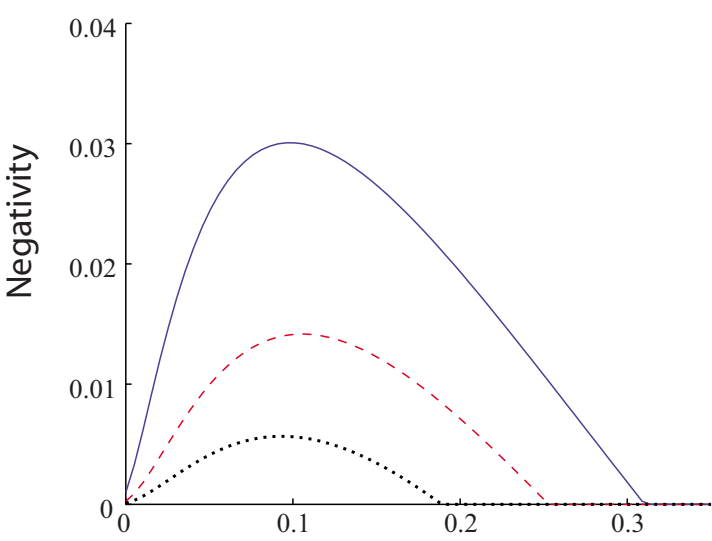

$\Lambda$

FIG. 5. (Color online) The steady-state negativity for our atomic system as a function of $\Lambda$, where we set $\Lambda_{1}=\Lambda_{2}=\Lambda, \gamma_{1}=\gamma=1, r$ $=1.2$, and $\gamma_{2}=r \gamma$. From bottom to top, the values of $\Gamma=0.8,0.9$, and 0.96 , corresponding to $R=1.18 \lambda_{1}, 0.83 \lambda_{1}$, and $0.5 \lambda_{1}$, respectively.

$$
\begin{gathered}
\rho_{68}=\frac{\gamma_{1} \Gamma_{2}}{s_{2} b}\left(\Lambda_{2}-\gamma_{2}\right) a_{1}, \\
\rho_{77}=\rho_{33}, \quad \rho_{88}=\rho_{66}, \\
\rho_{99}=\frac{2 \gamma_{1} \gamma_{2}}{b}\left(\beta_{1} a_{2}+a_{1} \beta_{2}\right),
\end{gathered}
$$

with

$$
\begin{gathered}
a_{1}=\Lambda_{2}\left[\gamma_{1}+2 \beta_{1}\left(\gamma_{1}+\gamma_{2}\right)\right], \\
a_{2}=\Lambda_{1}\left[\gamma_{2}+2 \beta_{2}\left(\gamma_{1}+\gamma_{2}\right)\right], \\
\beta_{j}=\frac{s_{\alpha} \gamma_{\alpha}^{2}+\Gamma_{\alpha}^{2}\left(\Lambda_{\alpha}-\gamma_{\alpha}\right)}{2 s_{\alpha} \gamma_{\alpha}\left(\Lambda_{1}+\Lambda_{2}\right)}, j=1,2, \\
b=2 \gamma_{1} \gamma_{2}\left(\gamma_{1}+\gamma_{2}\right)\left[\left(\beta_{1}+1\right) a_{2}+\left(\beta_{2}+1\right) a_{1}\right] \\
+\left(\gamma_{1}+\gamma_{2}\right)\left(a_{2} \gamma_{2} \Lambda_{1}+a_{1} \gamma_{1} \Lambda_{2}\right)+\gamma_{1} \gamma_{2}\left(2 a_{2} \Lambda_{2}+2 a_{1} \Lambda_{1}\right) .
\end{gathered}
$$

It is interesting to note that the steady state of the densitymatrix elements do not depend on $G_{1}$ and $G_{2}$. The level shift parameters $G_{1}$ and $G_{2}$ typically contribute to oscillation of population and coherence terms. In the steady-state limit, such oscillation terms vanish. Thus the above solutions in Eq. (17) are independent of $G_{1}$ and $G_{2}$.

Once again, as in the previous section, we calculate the eigenvalues of $\rho^{T_{A}}$ to measure the entanglement. We obtain equation for the nonzero eigenvalues as

$$
\begin{aligned}
& \left(\rho_{11}-\lambda\right)\left(\rho_{55}-\lambda\right)\left(\rho_{99}-\lambda\right)-\left|\rho_{37}\right|^{2}\left(\rho_{55}-\lambda\right)-\left|\rho_{68}\right|^{2}\left(\rho_{11}-\lambda\right) \\
& \quad=0 .
\end{aligned}
$$

We obtain the numerical values of $\lambda$ solving the above equation and substitute it in Eq. (12) to obtain the steady-state negativity as a function of $\Lambda_{i}$, as shown in Fig. 5. We have 
scaled the incoherent pumping rate $\Lambda_{i}$ with the spontaneous decay rate $\gamma_{1}=\gamma$ and also for simplicity we have assumed $\Lambda_{1}=\Lambda_{2}=\Lambda$. Clearly, a nonzero steady-state entanglement is obtained by incoherently repumping the excited state. The larger the value of $\Gamma$, the larger is the steady-state entanglement. Furthermore, as the incoherent pumping rate is increased the SGE increases but after reaching a certain optimal value at around $\Lambda \sim 0.08 \gamma$, the atomic entanglement starts to reduce. For smaller interatomic distances, even stronger incoherent pumping can be used to obtain SGE. Without any incoherent pumping the steady-state SGE vanishes.

Physically, the increase in entanglement with the incoherent pumping can be understood as follows: the spontaneous emission in either of the two atoms followed by exchange of photons between them generates SGE. But that does not survive long because the spontaneously emitted photon can escape in any arbitrary direction. Once both atoms loose their excitation, SGE vanishes. An incoherent pump assists the atoms to bring back the desirable excitation so that more spontaneous emissions and hence photon exchanges can take place between the two atoms. Thus, increasing the repumping via incoherent pumping helps increase the SGE. However, incoherent repumping also competes with the photonexchange process to re-excite the atoms. While an excitation due to the photon-exchange process enhances the entanglement, an excitation by incoherent process has no direct contribution to the entanglement. In fact, for a larger $\Lambda$, the incoherent excitation dominates the photon-exchange process and hence causes a decrease in SGE. For $\Lambda \gg \Gamma_{i}, G_{i}$, SGE becomes identically zero. This study clearly shows that, to generate maximum steady-state SGE between two atoms, there exists an optimal incoherent pumping rate which is determined by the $d d$ coupling parameters and decay parameters of the system.

\section{DISCUSSION AND SUMMARY}

We have investigated the spontaneously generated entanglement in a system of two three-level atoms that are coupled to a common vacuum field. We have presented time evolution of SGE due to the photon exchange between the two atoms. We have shown that both the magnitude of entanglement and the survival period of SGE are effectively enhanced by reducing the interatomic distance. From our analytical calculations, we have shown the strong dependence of the SGE on the radiative coupling parameters. We have demonstrated that the multilevel atoms are suitable for SGE due to availability of additional channels to establish entanglement. In the long-time limit, however, SGE vanishes.

Furthermore, to reinforce the above short term evolution of SGE in the radiatively coupled two-atom system, we have proposed using an incoherent pump that assists in repumping the deexcited atoms to generate a sustainable steady-state SGE. We have demonstrated that, for a certain range of incoherent pumping, the steady-state value of SGE increases as it prevents atoms from loosing their excited-state population. However, since incoherent pumping competes with the twoatom photon-exchange process to re-excite the atoms, a stronger incoherent pumping is shown to be undesirable. We have shown that an appropriate rate of incoherent pump can help in producing optimal SGE.

The radiative coupling discussed in this paper can be realized in a tight ion trap. However, this work can be generalized to realizing SGE in a chain of quantum dots or even in a typical dense multiatom system. We believe this work will open up a new way to utilize the naturally occurring SGE for realization of an efficient entanglement source. The above entanglement can further increase (not discussed here) if one considers atoms having degenerate or near degenerate excited states which has additional VIC [21]. We will discuss this situation elsewhere.

\section{ACKNOWLEDGMENTS}

A.K.P. is indebted to Professor G. S. Agarwal, Dr. P. Anisomov, and Professor M. O. Scully for discussions on various aspects of the two-atom multilevel systems. L.Z. thanks C. S. Yu for discussion on entanglement criteria. This work was supported by National Science Foundations (NSFC) under Grant No. 10774020, and also supported by Scientific Research Foundation (SRF), Returned Overseas Chinese Scholar (ROCS) program, State Education Ministry (SEM) of China.
[1] B. B. Blinov, D. L. Moehring, L.-M. Duan, and C. Monroe, Nature (London) 428, 153 (2004).

[2] Y. Miroshnychenko, W. Alt, I. Dotsenko, L. Fster, M. Khudaverdyan, D. Meschede, D. Schrader, and A. Rauschenbeutel, Nature (London) 442, 151 (2006).

[3] H. Haffner, W. Hansel, C. F. Roos, J. Benhelm, D. Chek-alkar, M. Chwalla, T. Korber, U. D. Rapol, M. Riebe, P. O. Schmidt, C. Becher, O. Guhne, W. Dur, and R. Blatt, Nature (London) 438, 643 (2005).

[4] S. Haroche and J.-M. Raimond, Exploring the Quantum (Oxford University Press, New York, 2006); M. B. Plenio and S. F. Huelga, Phys. Rev. Lett. 88, 197901 (2002); M. Skarja, N. M.
Borstnik, M. Loffler, and H. Walther, Phys. Rev. A 60, 3229 (1999).

[5] J. I. Cirac and P. Zoller, Phys. Rev. A 50, R2799 (1994); E. Hagley, X. Maitre, G. Nogues, C. Wunderlich, M. Brune, J. M. Raimond, and S. Haroche, Phys. Rev. Lett. 79, 1 (1997); M. B. Plenio, S. F. Huelga, A. Beige, and P. L. Knight, Phys. Rev. A 59, 2468 (1999); A. Beige, S. Bose, D. Braun, S. F. Huelga, P. L. Knight, M. B. Plenio, and V. Vedral, J. Mod. Opt. 47, 2583 (2000).

[6] G. K. Brennen, I. H. Deutsch, and P. S. Jessen, Phys. Rev. A 61, 062309 (2000).

[7] O. Mandel, M. Greiner, A. Widera, T. Rom, T. W. Hänsch, and 
I. Bloch, Nature (London) 425, 937 (2003).

[8] C. Cabrillo, J. I. Cirac, P. Garcia-Fernandez, and P. Zoller, Phys. Rev. A 59, 1025 (1999); O. Cakir, A. A. Klyachko, and A. S. Shumovsky, ibid. 71, 034303 (2005).

[9] M. Kiffner, J. Evers, and C. H. Keitel, Phys. Rev. A 75, 032313 (2007).

[10] L.-M. Duan, M. D. Lukin, J. I. Cirac, and P. Zoller, Nature (London) 414, 413 (2001); C. W. Chou, H. deRiedmatten, D. Felinto, S. V. Polyakov, S. J. van Enk, and H. J. Kimble, ibid. 438, 828 (2005).

[11] M. O. Scully, Laser Phys. 17, 635 (2007).

[12] M. O. Scully, E. S. Fry, C. H. Raymond Ooi, and K. Wodkiewicz, Phys. Rev. Lett. 96, 010501 (2006).

[13] S. Das, G. S. Agarwal, and M. O. Scully, Phys. Rev. Lett. 101, 153601 (2008).

[14] U. Akram, Z. Ficek, and S. Swain, Phys. Rev. A 62, 013413 (2000); L. Jakóbczyk, J. Phys. A 35, 6383 (2002); Z. Ficek and R. Tanaś, Phys. Rev. A 74, 024304 (2006).

[15] M. S. Kim, J. Lee, D. Ahn, and P. L. Knight, Phys. Rev. A 65, 040101(R) (2002); S. Schneider and G. J. Milburn, ibid. 65, 042107 (2002); J. Zhang and H. Yu, ibid. 75, 012101 (2007).

[16] D. Rossini, T. Calarco, V. Giovannetti, S. Montangero, and R. Fazio, Phys. Rev. A 75, 032333 (2007).

[17] L. Derkacz and L. Jakobczyk, Phys. Rev. A 74, 032313 (2006).

[18] Quantum Decoherence, Progress in Mathematical Physics, edited by B. Duplantier, J.-M. Raimond, and V. Rivasseau (Springer, New York, 2007), Vol. 48.

[19] Y. Li, J. Zhou, and H. Guo, Phys. Rev. A 79, 012309 (2009); J. Leon and C. Sabin, ibid. 79, 012301 (2009).
[20] J. P. Dowling and C. M. Bowden, Phys. Rev. Lett. 70, 1421 (1993)

[21] G. S. Agarwal and A. K. Patnaik, Phys. Rev. A 63, 043805 (2001)

[22] J. Evers, M. Kiffner, M. Macovei, and C. H. Keitel, Phys. Rev. A 73, 023804 (2006); M. Macovei, Z. Ficek, and C. H. Keitel, ibid. 73, 063821 (2006).

[23] M. Kiffner, J. Evers, and C. H. Keitel, Phys. Rev. A 76, 013807 (2007).

[24] M. Kiffner, J. Evers, and C. H. Keitel, Phys. Rev. A 75, 032313 (2007).

[25] L. Mandel and E. Wolf, Optical Coherence and Quantum Optics (Cambridge University Press, Cambridge, 1995).

[26] G. S. Agarwal, Quantum Statistical Theories of Spontaneous Emission and Their Relation to Other Approaches, Spring Tracts in Modern Physics: Quantum Optics (Springer-Verlag, Berlin, 1974).

[27] G. Vidal and R. F. Werner, Phys. Rev. A 65, 032314 (2002).

[28] K. Zyczkowski, P. Horodecki, A. Sanpera, and M. Lewenstein, Phys. Rev. A 58, 883 (1998).

[29] P. Horodecki, Phys. Lett. A 232, 333 (1997).

[30] The numerical parameters are obtained for a chosen geometry with $\theta=\frac{\pi}{2}, \phi=\frac{\pi}{4}$ in Eq. (26) of [21].

[31] O. Cakir, A. A. Klyachko, and A. S. Shumovsky, Phys. Rev. A 71, 034303 (2005).

[32] Y. Mu and C. M. Savage, Phys. Rev. A 46, 5944 (1992).

[33] T. Salzburger and H. Ritsch, Phys. Rev. Lett. 93, 063002 (2004). 